\title{
Apuntes sobre "Fuera y dentro del arte contemporáneo: comunidad y territorio en las prácticas colaborativas de Valparaíso"
}

Carol Illanes L. *

http://dx.doi.org/10.22409/poiesis.1829.027040

\begin{abstract}
RESUMEN: Entre el año 2013 y 2014 desarrollamos junto con Consuelo Banda una investigación de campo focalizada en la escena artística y cultural de Valparaíso, que tuvo por objeto las plataformas de trabajo colaborativo en esta ciudad. La investigación daría origen al libro publicado Fuera y dentro del arte contemporáneo: comunidad y territorio en las prácticas colaborativas de Valparaíso. En este texto propongo exponer de manera general las hipótesis desarrolladas en este trabajo, mencionando algunos contenidos presentes en la publicación. Esto, desglosando por un lado algunas de las complejidades a las que nos enfrentamos en el despliegue de los objetivos y por otro señalando puntos derivados de dichas hipótesis, basadas en una perspectiva contextual nacional.
\end{abstract}

PALABRAS CLAVE: Fuera y dentro del arte contemporáneo, comunidad y territorio, prácticas colaborativas

ABSTRACT: Between 2013 and 2014, together with Consuelo Banda, we developed a field research focused on the artistic and cultural scene of Valparaíso, which aimed at collaborative work platforms in this city. The research, which would give rise to the book published Fuera y dentro del arte contemporáneo: comunidad y territorio en las prácticas colaborativas de Valparaíso. In this text, I propose to expose in a general way the hypotheses developed in this work, mentioning some

\footnotetext{
${ }^{*}$ Carol Illanes L. es curadora, editora e investigadora independiente. Licenciada en Teoría y Historia del Arte en la Universidad de Chile. Cursando Magíster en Historia y Crítica de Arte en la Universidade Federal do Rio de Janeiro. Ha ejecutado diversos proyectos, publicado ensayos en revistas académicas y expuesto investigaciones en seminarios nacionales y internacionales sobre cultura y arte contemporáneo local. E-mail: carolillanes|@gmail.com.
} 
contents present in the publication. This, breaking down, on the one hand, some of the complexities that we face in the deployment of the objectives and, on the other, pointing out issues derived from these hypotheses, based on a national contextual perspective.

KEYWORDS: Fuera y dentro del arte contemporáneo, community and territory, collaborative practices

Entre el año 2013 y 2014 desarrollamos junto con Consuelo Banda una investigación de campo focalizada en la escena artística y cultural de Valparaíso, que tuvo por objeto las plataformas de trabajo colaborativo en esta ciudad. La investigación, que daría origen al libro publicado Fuera y dentro del arte contemporáneo: comunidad y territorio en las prácticas colaborativas de Valparaíso (ILLANES; BANDA, 2015), pretendía ir sobre tres objetivos: 1) realizar una radiografía de los espacios y agrupaciones en la localidad que han operado colectivamente en base al territorio en los últimos diez años, 2) armar un potencial relato en torno a ellos (incorporando disciplinas como la historia del arte, la historia urbana y la teoría crítica) y 3) inscribir y visibilizar una escena articulada por espacios basados en la autogestión, también sintomática de los modelos de producción artística regional, analizando sus posibles causas y consecuencias en el campo cultural y social. En definitiva, lo que se planteó era la observación a las infraestructuras artísticas creadas a pulso que pronto veríamos terminan (o empiezan) siendo atravesadas por una "tradición" colaborativa de la región. ¿Por qué Valparaíso? Porque su historia reciente la posiciona como una ciudad ejemplarmente sobreexpuesta a las violencias desatadas por los procesos de modernización, la especulación del territorio y la sobreexplotación de una imagen cultural patrimonial. De allí la hipótesis principal del libro:

las políticas de Estado y su respuesta ciudadana hicieron de Valparaíso un lugar propicio para prácticas desmarcadas de la institucionalidad oficial, dando lugar a un escenario donde el trabajo colectivo con énfasis en el territorio y la comunidad se presenta como fin y modelo predominante. Esta escena -suspendiendo la pregunta de si es posible incluso llamarla como tal -, si bien no es homogénea comparte una orientación y representa un caso paradigmático de la relación arte y vida en la actualidad, en tanto escala y naturaleza del involucramiento que estas prácticas tienen con la esfera social. (ILLANES; BANDA, 2015, p. 10) 
Así, la publicación se resolvió en dos partes. La primera compuesta por tres ensayos es, podríamos decir, de "perspectiva". En ellos se recorren algunos referentes, esbozando un devenir histórico con una apreciación de los objetos de estudio en un marco ampliado -particularmente en tres asuntos: una meta lectura de la publicación como "síntoma"; la relación local entre arte en espacio público y crítica institucional; y la historia de la ciudad de Valparaíso en forma de cuatro "ficciones". Una segunda parte presenta a cada plataforma de manera independiente, mediante un formato más cercano al del catálogo (proponiendo definiciones en base a perfiles: comunitario, formativo y de producción específica).

En este texto propongo exponer de manera general las hipótesis desarrolladas en este trabajo, mencionando algunos contenidos presentes en la publicación. Esto, desglosando por un lado algunas de las complejidades a las que nos enfrentamos en el despliegue de los objetivos y por otro señalando puntos derivados de dichas hipótesis, basadas en una perspectiva contextual nacional.

\section{La inventiva de la mirada}

A diferencia de la crítica de arte y cierto campo de la curaduría que asumen su naturaleza sincrónica como "fracaso productivo," la historia y la teoría del arte deben convivir con dificultades de orientación al poner la atención en fenómenos muy recientes, cuestión que auxilia -a la vez que deslegitima- el campo de decisiones metodológicas e interpretativas del objeto en cada caso.

En este sentido, este trabajo dejó, natural y, podríamos decir, afortunadamente, más preguntas que respuestas, principalmente por las dificultades de circunscribir una escena que se ampliaba en su observación (implicando el involucramiento de campos extra artísticos con los cuales reconocíamos una serie de limitaciones). Digo afortunadamente, porque la desorientación inicial se subsumía también a la relativa carencia de iniciativas teóricas de este tipo en el contexto local, lo cual permitió asumirla como un primer paso, una primera mirada a un fenómeno cuyas proyecciones excedían el marco específico del arte urbano y el arte contemporáneo. 
Este asunto, el que dicho espectro demarcado desde su inicio se volviera dificultosamente abarcable en términos disciplinares y conceptuales, planteó una primera alerta para con lo que pretendíamos cartografiar. Traduzco esto mediante dos ejemplos concretos. El primero atiende la decisión de incorporar el Festival de Arte Sonoro Tsonami, la plataforma focalizada en las artes escénicas Escenalborde y el colectivo artístico Pia Michelle. Estas son plataformas de "producción específica", generadas en base a una diagnosticada necesidad de constituir espacios de experimentación (tras la desidia institucional y académica), con un diálogo clave con el espacio público. Si bien estos espacios no dependen de un lugar específico, un espacio físico que componga su identidad como proyecto, son iniciativas que destacaron rápidamente por su vinculación al territorio, la sostenida investigación en torno a la ciudad y la preocupación por las mediaciones de la producción en las áreas de la danza, el teatro, el arte sonoro y las artes visuales respectivamente.

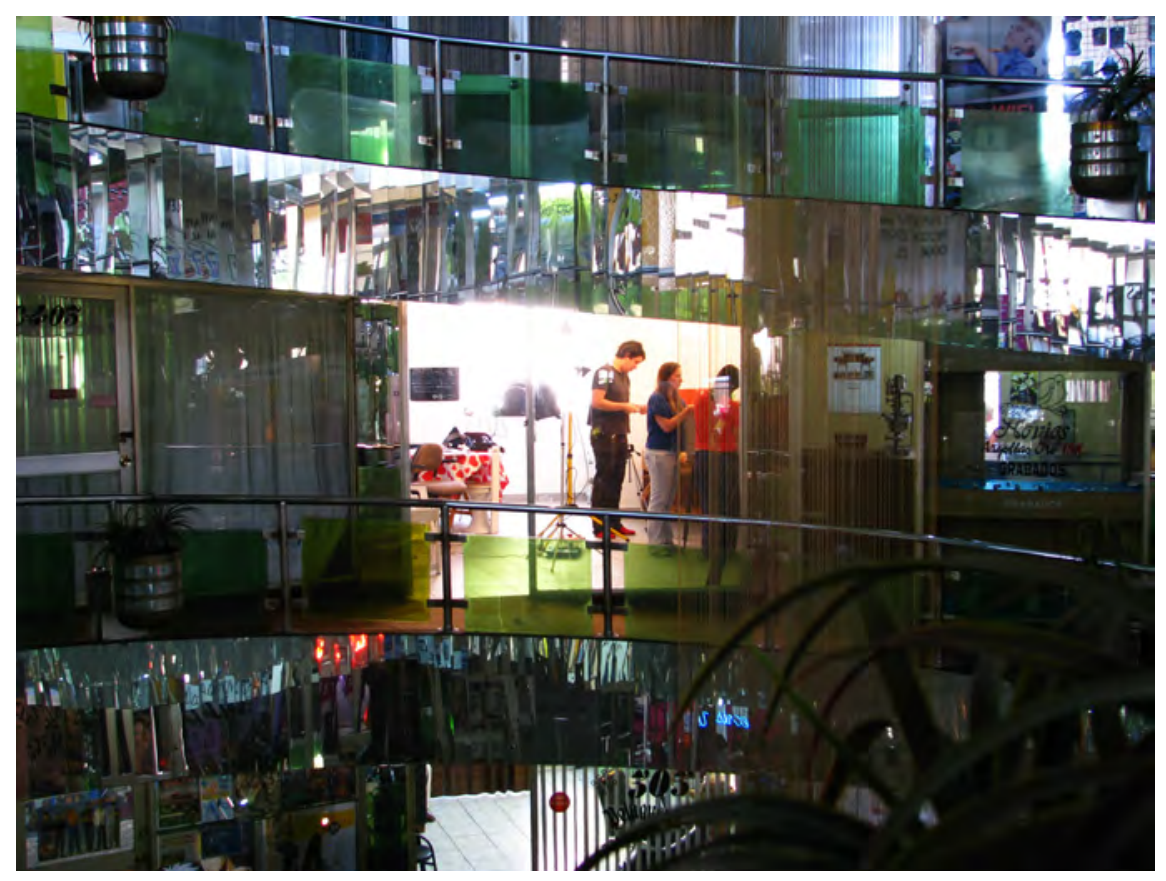

Pia Michelle, espacio del colectivo, vista interior edificio Centro Cultural Tres Palacios, 2009, cortesía Pia Michelle. 
El proyecto principal de Escenalborde, el Festival Danzalborde, fue por ejemplo el primero en combinar muestras de sala con intervenciones urbanas, una línea que se ha ido consolidando con los años, siendo parte fundamental de su propuesta en tanto relaciona una investigación en torno al cuerpo con la arquitectura, y con ello diferentes formas de exploración de uno de los grandes temas de la ciudad, el patrimonio. Por su parte, Tsonami ha fomentado la socialización y reflexión de las prácticas sonoras contemporáneas (conciertos, actividades de formación, etc.) donde las operaciones y obras site-specific fortalecen y cumplen un lugar fundamental. Por su parte, en una menor escala pero significativa dentro de esta línea, el colectivo Pia Michelle (en la línea de lo que fue años atrás la galería vitrina h10) propuso la salida y exposición de los procesos de producción de la investigación artística, interviniendo un espacio dentro de una galería comercial.

No siendo menor el que sean propuestas - muchas veces obligadamente - autogestionadas, nacidas por las insuficiencias del aparato institucional regional y académico, estas iniciativas se proponen hacer dialogar una producción particular con instancias de formación y mediación (talleres, obras participativas, etc.), abocándose a pensar los problemas de la ciudad de Valparaíso. Son plataformas que eligen "salir" de su campo específico para ampliar el espectro receptivo a un público general, llevando proyectos concretos a la ciudad.

Nos pareció primordial incorporar estos casos, no solo por la vocación pública y urbana que veíamos operaba en ellos, sino también por como ellas van logrando la conquista de espacios que acrecientan los terrenos de impacto. El proyecto editorial de la revista Aural en Tsonami, la proyección curatorial de Pia Michelle, las intervenciones masivas participativas de Danzaborde, son algunas de las salidas que van explorando otros formatos de mediación que ciertamente colaboran a repensar cómo se genera en lo concreto la preocupación por la ciudad contemporánea en el arte. Son plataformas, entre muchas otras, que promueven de forma explícita una "puesta en obra" de dicha preocupación. Es allí donde gestión, arte en espacio público y disciplinas artísticas parecen tocarse de una manera particular en este contexto, que creo incita precisamente a expandir la mirada, debido a que los espacios especializados en arte contemporáneo (tratados como tal en esta publicación también), se sirven de estrategias que están "por fuera" de su campo de acción, relacionándose más a estos modelos. Menciono este ejemplo, aparentemente aislado del tema inicial del libro, solo para 
ejemplificar lo provisorio de los límites de una investigación que en un principio se planteó para pensar el "arte colaborativo"; el contexto toma la palabra, incidiendo en las decisiones de abordaje que en este caso pusieron en tensión los límites de las prácticas y su asimilación o misma administración teórica.

\section{Los difusos lindes de "lo público" en el arte}

La categoría del arte público ha convivido con el carácter difuso de su propio nombre; su amplitud es desbordante. Y aunque arte, ciudad y práctica experimental (de intervención, performática o de acción) acortan a medio siglo sus referentes, lo cierto es que el activismo, la escultura, el cuerpo, la comunidad, entre otros grandes conceptos o marcos de reflexión, ingresan en el relato del arte contemporáneo elaborando cada uno su propia historia. Las prácticas colaborativas estarían insertas dentro de este problema. Como define el glosario de Redes Instituyentes ${ }^{2}$, estas se focalizan en los modos de colaboración y negociación entre trabajadores culturales y diferentes colectivos y redes sociales, que generan procesos y transformaciones en un grupo social (de variada escala), tras una proyección temporal de trabajo. En él se comparten temas en común a las partes, siendo fundamental pensar el tipo de intercambios producidos.

Queda claro que lo central es entonces una forma de relación, un "modo de hacer". Cuando pensamos esto, las categorías de arte público del nuevo género o arte público comunitario tienden a ser insuficientes para pensar la expansión de del concepto de comunidad en ciertos proyectos artísticos. Hoy, como dice Alfredo Palacios todo ha tendido a entremezclarse " [...] y las fronteras entre arte público, arte comunitario, educación e intervención social a ser menos visibles" (Palacios, 2009: 4). Esta sentencia, que apronta la dificultad conceptual señalada, es posible verla concretamente en el trabajo realizado en el libro. En el caso del "perfil de producción específica", por ejemplo, conviven registradas amplias plataformas de trabajo en red como CRAC Valparaíso (centro de residencias que hoy coordina proyectos bajo el modelo de las pedagogías colectivas) con microgalerías como La Pan (galería experimental que se invita a intervenir, dispuesta en una panadería), o espacios como Worm Gallery, Casa Nekoe, Gálvez Inc., galerías "domésticas" entregadas al barrio, que se transformaron en espacios de exposición de arte contemporáneo. 


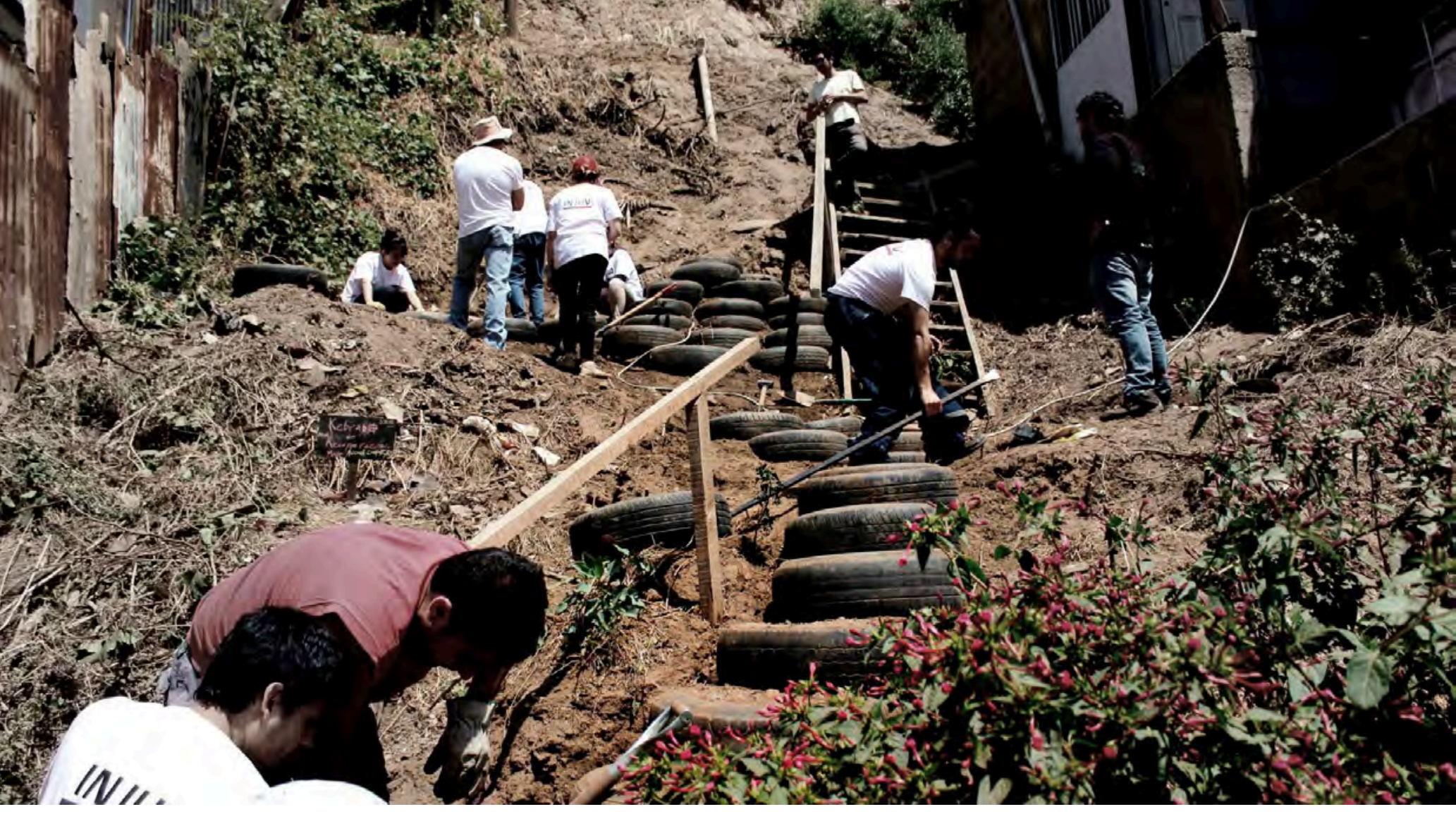

Trabajos quebrada cerro La Loma, Áncora, 2015, cortesía Áncora

Por otro lado, dentro del "perfil comunitario" aparecen lógicas como las de Patio Volantín, una casa que funciona en base al intercambio y las cooperatividad y Áncora, un espacio cowork abierto a la comunidad creativa (dentro este seguimiento implicó explicar varios antecedentes como el Taller de Acción Comunitaria, el Centro Comunitario las Cañas, Centro Cultural Trafón y el Espacio Santa Ana, cruciales en la coyuntura de Valparaíso post-incendio).

Tras la incorporación de casos aparentemente disímiles pero conectados a su trabajo con comunidades específicas una pregunta crucial emergió de forma inmediata ¿Cómo es que "lo público" (particularmente la dimensión pública de la ciudad y los problemas urbanos) en el arte se vuelve "inmanente" en una ciudad como Valparaíso? 


\section{Las aceleraciones de la ciudad}

Esto lleva a señalar un tercer punto, la necesidad de incorporar la historia de la ciudad. Como se insiste en el libro cuando se describe la ciudad de Valparaíso se tiende a utilizar el concepto de resiliencia. Una historia de deficientes administraciones y crisis político-económicas hablan de una ciudad construida en base a imposiciones y supervivencias, en definitiva, de improvisaciones, haciendo frente a esa otra fuerza que intenta configurarla como imagen turística, como identidad estática:

Esta noción obstinada del puerto como riqueza, como lugar de transacciones y aperturas económicas, es la misma noción que renueva e improvisa ficciones para la ciudad. Dichas ficciones, crean la imagen de que, frente a cualquier adversidad, a cualquier mega-construcción o cualquier política instaurada, la ciudad se recoge y se repone, contra viento y marea, para asumir y asimilar todo cambio de planes que públicos y privados puedan determinar. (ILLANES; BANDA, 2015, p. 66)

La compleja geografía del puerto (plan/cerro), la sectorización radical de los terrenos de comercio, turismo e inversión inmobiliaria de los cerros patrimoniales se contrapone a los extremos invisibilizados. Expuestas como la encarnación de esta naturaleza resiliente del desarrollo urbano del puerto, se trataron cuatro "ficciones" en base a tres enunciados históricos: Valparaíso Patrimonio de la Humanidad, Valparaíso Capital Cultural y Valparaíso Puerto principal.

Valparaíso no ha dejado de sufrir los efectos de la proclamación del Casco Histórico como patrimonio por la UNESCO el año 2003. La persecución de este título, que suponía potenciar a la ciudad como polo turístico (particularmente la arquitectura colonial extranjera del XIX), sin las políticas de restauración y conservación idóneas, se transformó en la "impronta del revestimiento", una noción estética de lo patrimonial, descuidando el campo simbólico o inmaterial como las costumbres, oficios, la vida en el puerto. Pese a las grandes expectativas -Valparaíso tenía los índices de cesantía más altos a nivel nacional en ese entonces- y a la especulación sobre las oportunidades que los habitantes del puerto tendrían gracias a la nueva inversión pública y privada, rápidamente se visibilizaron los problemas gubernamentales y municipales, la inexistencia de una competencia institucional para concretar metas a corto plazo, y la falta de coordinación entre el gobierno regional, municipal y el gobierno nacional, imponiendo planes reguladores y administraciones desprovistos de coherencia interna. 
La segunda ficción, la de la ciudad como Capital Cultural, toma forma en la figura de los Carnavales Culturales (2001-2010), el emblema de las nuevas políticas culturales, basado en el modelo de entretenimiento y recreación mediante eventos que pretenden evocar la tradición de la fiesta callejera. Tras el traspaso al Consejo Nacional de la Cultura y las Artes, se somete al evento a una serie de programaciones, haciéndose latente el malestar por la falta de participación ciudadana, cuestión que se agrava tras la transformación de los Carnavales en el Festival de las Artes bajo el gobierno de Sebastián Piñera. Será inmediato el malestar de las organizaciones culturales y la opinión pública que concibe esta "elitización" como manifestación de la desidia por parte del gobierno hacia sector cultural, un intento de borrar el referente del carnaval para someterlo a una higienización de las manifestaciones de carácter popular (mientras los Mil Tambores se convertirán en su versión antagónica, resistencia de la "cultura popular").

La tercera ficción, la de Valparaíso como Puerto Principal, atendió el drama de las luchas por el borde costero, particularmente el proyecto de construcción del Mall Barón que dio pie a una serie de conflictos y manifestaciones sociales. Acá el problema de gentrificación apareció con toda violencia, y con inmediato eco en las organizaciones ciudadanas de defensa del territorio. Pese al estanco del proyecto -que a pesar de su opositores contaba con el apoyo de la alcaldía, la Empresa Portuaria (EPV), el Ministerio de vivienda y parte de la Unión comunal de Juntas de vecinos-, luego de diez años siguen reverberando los peligros entorno al destino del borde costero.

La ficción final atiende el proceso de creación y construcción del Parque Cultural de Valparaíso, anterior Centro Cultural Ex-Cárcel. Un espacio de actual disputa donde aparece la organización comunitaria, la oposición institución/ciudadanía y la activación de un circuito artístico devenido del periodo de cierre y reestructuración del Parque. Es este acontecimiento cultural, la transformación y revitalización de un espacio inhóspito gracias a sistemas comunitarios, pero luego arrasado por la implantación de un modelo de imagen-ciudad, el que daría

pie a la activación de un circuito cultural local que trasciende los límites de la cárcel. Podemos ver este proceso como un catalizador de prácticas y estrategias de reapropiación, a partir de la creación de nuevos centros culturales esparcidos en el territorio, los cuales corresponden a diversos grupos, colectivos e iniciativas que de alguna manera tuvieron una relación y comunicación con los procesos vividos en la Ex Cárcel. (ILLANES; BANDA, 2015, p. 87)

Con una historia urbana como Valparaíso, pensar estos fenómenos se vuelve crucial por su impacto en el desarrollo de los movimientos sociales y la respuesta ciudadana. 


\section{Preocupación epocal por las mediaciones}

En diciembre del año pasado se publicó en Chile el libro La puesta a prueba de lo común. Una aproximación a los discontinuos trazos de la dimensión colectiva en el arte contemporáneo penquista. (MUÑOZ; ROMERO, 2014) En él los autores David Muñoz y Cristián Romero, fundadores de la Revista Plus, revisaron las transformaciones del arte del último tiempo en la ciudad de Concepción, desglosando el panorama del trabajo colaborativo en el contexto local, de fines de los años noventa y principios del 2000. Los modelos de trabajo colectivo, las experiencias experimentales en el espacio público, las insuficiencias del aparato institucional, fueron los temas tratados en este libro, colaborando al reciente debate nacional sobre la importancia de estos "otros" contextos, fuera de la capital, que se han hecho visibles en los últimos años. Este libro, junto a un conjunto de propuestas locales en la misma línea, venía a ilustrar una preocupación que pronto se consolidaría como diagnóstico.

Este debate, donde aparecen conceptos ligados al estatuto de las plataformas -"autogestionado", "autónomo," “independiente," “autodependiente"- y la mediación con el contexto social -"esfera pública", "comunidad"-, toman lugar no solo gracias al espíritu descentralizador (urgencia ya instalada), que sus propios protagonistas ejercen al exponer sus modelos de trabajo, sino también al interés gubernamental que estas propuestas comienzan a tener en las escenas locales. El gesto de observación y seguimiento por parte del Estado de estas iniciativas, síntoma de diversas carencias en las políticas culturales, se corresponde a un escenario político-económico que evidencia un cambio en las estrategias de "resistencia" de procesos creativos de corte experimental o sin fines comerciales; uno donde las propuestas ya no pueden permanecer en la marginalidad y deben imbricarse con campo institucional, los organismos de Estado y la iniciativa privada.

Uno de los asuntos que se destaca transversalmente en los espacios que generan prácticas colaborativas en el contexto estudiado, es el de incorporar instancias formativas; en la última década, el campo artístico en general exhibe la reiteración de términos asociados a la "democratización" y la "mediación". En este sentido, es posible asociar el fenómeno de las prácticas colaborativas a una transformación de mayor escala, relacionada con el resurgimiento de diferentes manifestaciones sociales (inicialmente asociadas a los problemas en la educación chilena) en el contexto local y su influencia en la evaluación del rol del Estado en la cultura. 


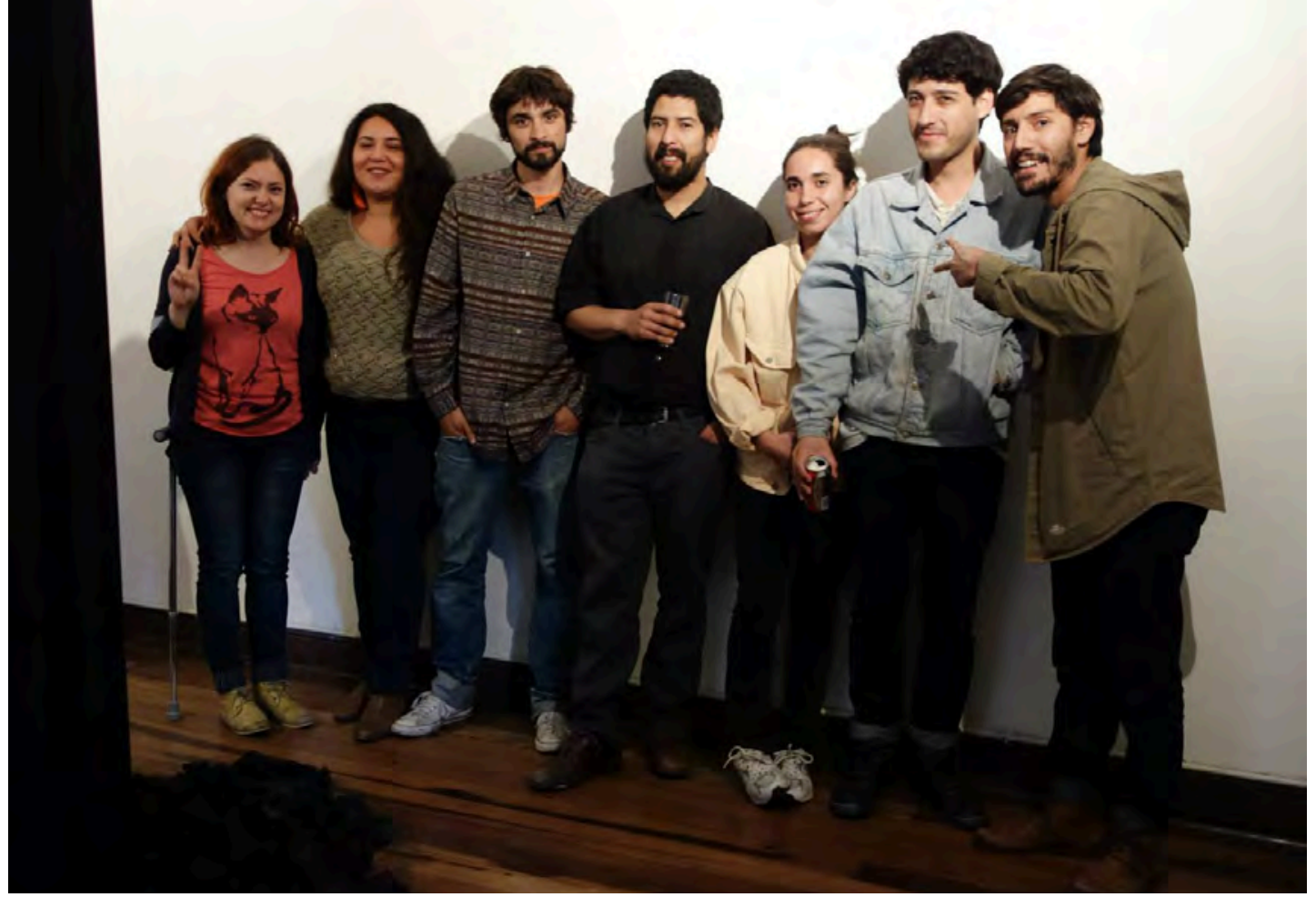

Residencia Casa Nekoe, Gustavo Arenas, 12 diciembre 2014, Grupo Worm, cortesía Casa Nekoe

La pregunta extendida hacia el rol del arte contemporáneo -expresada inéditamente en los medios de comunicación y la esfera pública luego, por ejemplo, del surgimiento de un arte de corte activista-, forma parte de este debate así como también el progresivo desarrollo de líneas formativas en las áreas de extensión de instituciones "oficiales". Es este sentido, observar la emergencia de prácticas ligadas a la comunidad puede hablarnos de transformaciones de carácter más profundo en nuestro contexto.

\section{Crítica institucional como factor constituyente}

La herencia de la neovanguardia chilena ha calado hondo en el concepto de "arte político" en nuestra historiografía. Sin embargo, la mirada hacia el fenómeno de las prácticas colaborativas ha ayudado a descentrarla hacia otras formas de relación de lo político en el arte. 
Dos cuestiones afloraron en relación a este problema. Un primer asunto refiere a la confirmación del lugar protagónico de la figura de la crítica institucional en el arte local y a las características particulares que se exhiben en su emergencia en Valparaíso. Esto permitió elaborar un contraste inmediato entre el contexto metropolitano y el porteño. El rol de las instituciones académicas en Chile se ha asimilado gracias a sus efectos visibles, sin embargo, está en deuda la observación a locaciones cuyas condiciones se presentan más adversas que en el conocido escenario metropolitano. "Si el primero se ha mostrado como el escenario representativo de la inherencia académica y la institucionalidad oficial desde donde emerge el grueso de la producción crítica; en el segundo tendríamos un escenario donde ésta tiende a ocurrir 'por fuera' de ella o en respuesta a sus atrasos, carencias y precariedades". (ILLANES; BANDA, 2015, p. 170)

Un segundo asunto refiere a las estrategias de la crítica institucional en la expansión del arte al espacio público (asunto transversal en los objetos de esta investigación). En el contexto porteño estas estrategias se ven caladas claramente por los problemas urbanos, ante una ciudad que lucha con su especulación. La respuesta de la cultura se hace presente en la "intemperie", la que es intervenida y apropiada.

En este sentido, iniciativas como la galería vitrina h10 a comienzos de los 2000, viene a encarnar una crítica que se sostendrá hasta el presente. Mínimos recursos, amplias voluntades. El problema de la carencia de los espacios de exhibición y la necesidad de autosustentar proyectos de manera autónoma en contextos donde la institucionalidad cultural local carece de presencia. Un antecedente fundamental y fundacional a la alerta de la precariedad de circulación de arte contemporáneo en la ciudad.

\section{El peligro asimilado de la cooptación}

Siguiendo lo anterior, Valparaíso está permeado por su intento constante de responder al modelo de las industrias culturales. Sin embargo, la mayoría de los agentes asume la dificultad de sostener modelos que puedan alejarse totalmente de las vinculaciones con instituciones oficiales o el mercado. La figura recurrente es la de la paradoja, en la medida en que el aislamiento al sistema cultural no corresponde a un régimen neoliberal que cala absolutamente toda las estructuras. Sin embargo, el problema se acrecienta cuando se le suma el progresivo 


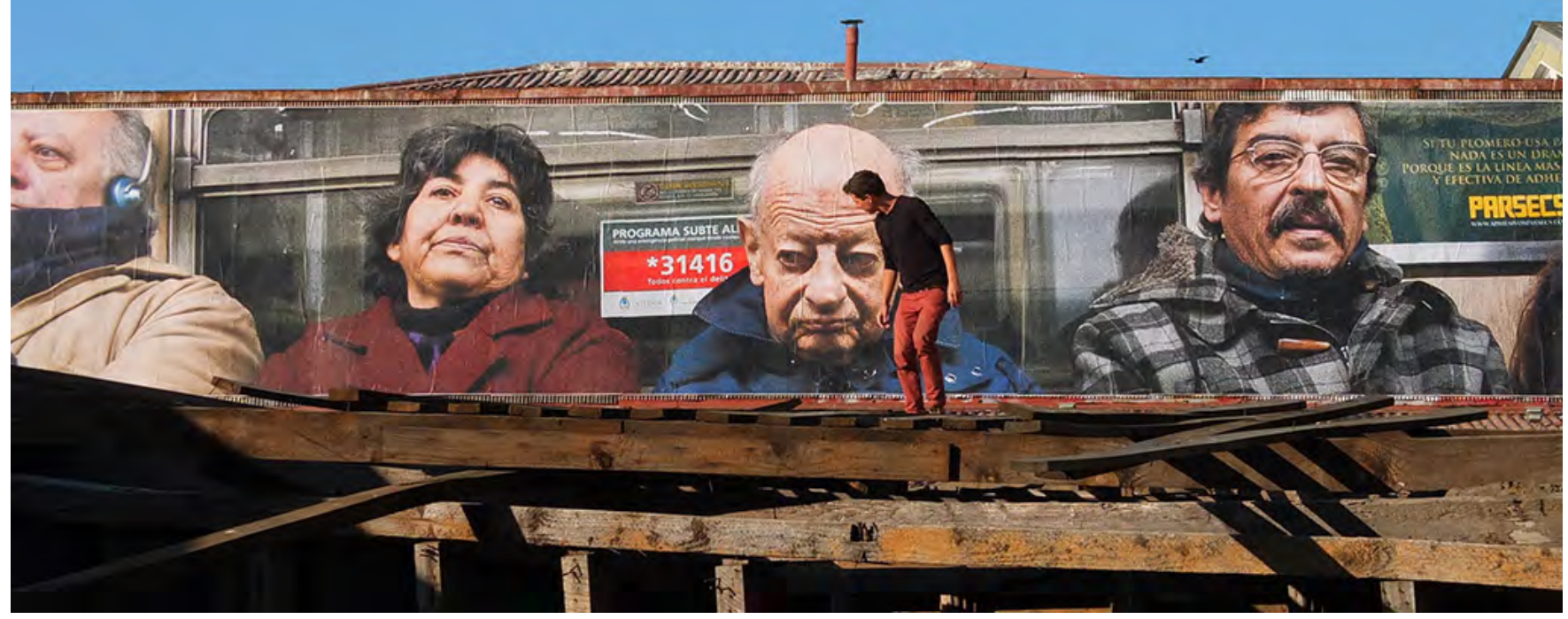

Gálvez Inc., Registro Walltoheaven, obra de Arnaldo Utrera, cortesía Gálvez Inc.

interés por parte de los organismos de Estado en la incorporación tardía de estas retóricas nacidas "en los márgenes" (por mencionar un ejemplo, en agosto del 2014 el Centro de Extensión del CNCA inaugura la Fábrica Puelagalán "Colaborar+Crear+Reutilizar" intentando proponer una instancia que hiciera ritmo a la serie de medidas autogestionadas por la propia ciudadanía tras el incendio. El modelo era aportar a la reconstrucción de hogares afectados tras la catástrofe de abril a partir de técnicas textiles, culinarias y talleres de diversa índole en un espacio de exposición).

El "fuera y dentro" en este sentido habla de la astucia obligada de los agentes culturales (entrar y salir de la institución, dice Marcelo Expósito). Como finaliza el libro

Ante las paradojas con las que el arte "resistente" debe convivir es necesario no solo la propuesta de nuevas categorías y formas de comprender las relaciones con el campo extra-artístico sino también interiorizar sus limitaciones dentro de un actual escenario de contradicciones. En el caso del arte que trabaja con la comunidad comprender que este no estaría exento de colonizaciones, paternalismos o reificaciones, lo que implica reconceptualizar constantemente aquella utopía que todavía llamamos comunidad. (ILLANES; BANDA, 2015, p. 171) 


\section{Notas}

1 Este texto fue publicado en el libro Pasados presentes. Debates por las memorias en el arte público en Latinoamérica. Cali: Universidad del Valle - GEAP Latinoamérica, 2015. ESPANTOSO R., Teresa; MUÑOZ, Carmen C.; RECIO, Carlos M.; VANEGAS, Carolina C. (eds.) a propósito del IV Seminario Internacional sobre Arte Público en Latinoamérica.

2 GLOSARIO "REDES INSTITUYENTES" [en línea] http://redesinstituyentes.wordpress.com/glosario-y-referentes [consulta: 20 mayo 2014]

\section{Referencias}

ARAVENA, Pablo. Patrimonio, memoria e historicidad: El contenido político de nuestra relación con el pasado. (2005) [en línea] http:// www.archivochile.com/Ceme/recup_memoria/cemememo0042.pdf

EXPÓSITO, Marcelo. Entrar y salir de la institución: autovalorización y montaje en el arte contemporáneo. (2006) [en línea]: http:// eipcp.net/transversal/0407/exposito/es

GARCÍA FERNÁNDEZ, Isabel. ¿La nueva crítica institucional? (2010) [en línea]: http://ler. letras.up.pt/uploads/ficheiros/10365.pdf

GARRIDO, Alfredo Palacios. El arte comunitario: origen y evolución de las prácticas artísticas colaborativas. En Arteterapia. Papeles de arteterapia y educación artística para la inclusión social, v. 4, p. 197-211, 2009.

ILLANES, Carol; BANDA, Consuelo. Fuera y dentro del arte contemporáneo:

Comunidad y territorio en las prácticas colaborativas de Valparaíso. Santiago: Adrede Editora, 2015.

KWON, Miwon. One Place after Another: Site Specific Art and Locational identity. Cambridge: The MIT Press, 2002.

RANCIÈRE, Jacques. Las paradojas del arte político. En El Espectador emancipado, Buenos Aires; Bordes Manantial, 2010.

RAUNIG, Gerald. La industria creativa como engaño de masas. En VVAA, Producción cultural y prácticas instituyentes. Líneas de ruptura en la crítica institucional. Madrid: Transform, Traficantes de sueños, 2008, p. 27-42.

SEPÚLVEDA, Sebastián. Barrio, Comunidad y Patrimonio Cultural Un estudio cualitativo sobre los habitantes de los cerros Alegre y Concepción de Valparaíso. Santiago: Universidad de Chile, 2008. 